\section{La Problematización En Investigación Educativa Desde La Depuración Sucesiva De Mapas Conceptuales}

\title{
Resumen
}

En los cursos de seminario de maestrías relacionadas con educación, se ha detectado que los textos sobre el planteamiento del problema de investigación de los docentes noveles investigadores, generalmente no presentan coherencia, claridad en el objeto de estudio, ni argumentos adecuados. Este estudio tuvo como propósito indagar sobre cómo puede ayudar una estrategia basada en mapas conceptuales sucesivos depurados, a los docentes a clarificar su problema de investigación. Para el desarrollo de la investigación se diseñó e implementó una estrategia basada en la construcción y depuración de tres mapas conceptuales sobre: un tema de la maestría, qué es un problema de investigación y del problema de investigación del docente. Este estudio se desarrolló bajo un enfoque cualitativo y el método dialógico crítico. La experiencia se hizo con cinco docentes y los instrumentos de recolección de información fueron: las grabaciones de audio de los diálogos recursivos entre el docente y el tutor/asesor, y los mapas conceptuales sucesivos depurados. Entre los hallazgos destacan: la emergencia de una categoría asociada a los estados emocionales de los participantes, y que la estrategia propuesta disminuye los rasgos de borrosidad de los docentes investigadores y resulta promisoria para ayudarles a clarificar su problema de investigación.

Palabras claves: el problema de investigación, investigación educativa, mapa conceptual sucesivo depurado, dialogo recursivo.

\begin{abstract}
In the seminary courses of master degrees related to the education area, has found repeatedly the absence of some important elements in the research texts produced by those teachers, who start in the path of investigation, these elements are coherence, clarity in the object of study, or adequate arguments. This study aimed to investigate how a strategy based sequential debugged concept map can help teachers clarify their research problem. For the development of the research, a strategy based on the construction and debugging of three concept maps was designed and implemented on: a subject of master degrees, what is a research problem and the teacher's research problem. This study was developed under a qualitative approach and the critical dialogic method. The experience involved five teachers enrolled in a master program, and the instruments for collecting data were the audio recordings of the recursive dialogues and the sequential debugged concept map. The findings include: the appearance of a category related to the emotional states of the participants, and the proposed strategy minimizes the diffuse performance of the new researchers and at the same time, this strategy is promising to help them clarify their research problem.
\end{abstract}


Key words: the research problem, educational research, sequential debugged concept map, recursive dialogue.

\section{Introducción}

La investigación educativa debe ser abordada por los docentes en algún momento de su vida académica, y se entiende cómo la búsqueda de respuestas sistematizadas y metodológicamente científicas a algunas de las innumerables interrogantes que pueden surgir en su práctica como docente. El acto educativo es complejo (Sánchez, 2014) o como lo diría este mismo autor "altamente complejo", y el docente investigador se ve en la necesidad de planificar su acción para identificar problemas y plantear objetivos de investigación, así como también perfilar caminos metodológicos conducentes al logro de estos objetivos, seleccionar los medios más apropiados, hacer procedimientos, tomar decisiones, retroalimentar el trabajo y evaluar entre otras labores.

En la Universidad Nacional Experimental del Táchira (UNET) se ha detectado que a estudiantes de postgrado de maestrías en educación, docentes investigadores noveles, se les dificulta el desarrollo de su proceso investigativo. Esto se evidencia al revisar la cantidad de deserciones en los postgrados debido a la no culminación de su trabajo de grado producto de un proceso investigativo y en otras latitudes la situación es similar, reportándose en los postgrados altos niveles de abandono básicamente por la misma razón (Ochoa y Cueva, 2012).

El docente novel investigador ante el amplio panorama de información existente sobre investigación y los temas sobre educación, al iniciar un proceso de investigación educativa, se encuentra en el centro de una gran cantidad de opciones sobre temáticas problematizadoras, posturas teóricas y metodológicas con la inquietud de decidir cuál recorrer, lo que le genera una alta carga emotiva a manejar. Por ello debe desarrollar habilidades para la organización de sus ideas que le permitan reflexionar y autorregularse y las dificultades que enfrenta pueden originarse por múltiples causas, entre las cuales resaltan: (a) limitadas competencias para la lectura y escritura académica (b) limitadas competencias investigativas; (c) desconocimiento de los conceptos propios de la maestría y procesos metodológicos; (d) ausencia de estrategias metodológicas en los procesos de tutorías o asesorías (Moreno, 2016; Najera, Murillo y García, 2017; Perines, 2018).

En el Congreso Internacional de Mapas realizado en Brasil, Sanabria, Ramírez, Téllez y Aspée (2014) reportaron una experiencia metodológica con enfoque cualitativo, realizada con un grupo de 10 docentes para orientar el desarrollo de una investigación utilizando mapas conceptuales sucesivos depurados (MCSD). Los participantes desarrollaron sus trabajos bajo la tutoría de integrantes del grupo de investigación del que forman parte los autores. La estrategia metodológica consistió en el uso de mapas conceptuales sucesivos depurados durante las distintas etapas de la investigación. En usos posteriores se encontró que esta estrategia funciona exitosamente con los docentes investigadores que tienen claridad en su tema de investigación. Sin embargo, al planificar acciones para la incorporación de esta estrategia en los cursos de Seminario se encontraron también limitaciones en cuanto al tiempo (48 horas académicas), la cantidad de participantes en estos cursos (alrededor de 20), y la falta de claridad en los participantes en cuanto al tema de investigación y el proceso investigativo.

La autora principal de este trabajo como profesora de Seminario I, en postgrado, durante 4 cohortes (desde el 2016 al 2017) detectó que a los maestrantes se les dificultaba definir objetos de estudio y concretar problemas de investigación. Las estadísticas del trabajo de los maestrantes arrojaron que el $80 \%$ de ellos en estos cursos de Seminario, no logró precisar su planteamiento del problema. En ese primer intento de plantear un problema de investigación, muchas veces, no se clarifica el problema, el cual (como resultado de estos cursos) resulta desarticulado, descontextualizado, difuso y sin 
argumentos; a lo que se suma el estado de inquietud del maestrante por su escasa o inadecuada producción.

Los cursos de metodología de la investigación, generalmente se dirigen a la estructura, contenido y formato de los proyectos de investigación, y su metodología se centra en la propuesta de un conjunto de pasos secuenciales, realizados presurosamente para cumplir con un cronograma. Se comparten las ideas de Sánchez (2014) de que los cursos de metodología ofertados por las universidades no deben ser rígidos en cuanto a contenidos, orientaciones epistemológicas y metodológicas, por el contrario, deben ser flexibles, adecuados a cada ciencia, disciplina y a las necesidades de formación del investigador novel. Este tema ha sido ampliamente trabajado por autores como Ochoa (2011) y Valarino (1997) cuyas recomendaciones orientan a estudiarlo y profundizarlo desde factores tanto internos como externos a los investigadores noveles y proponer acciones metodológicas contextualizadas y adaptadas a la naturaleza de los temas propios de las maestrías.

Ante esta realidad se plantea la necesidad de desarrollar posibles estrategias que permitan a los maestrantes (docentes noveles investigadores) identificar, clarificar y definir un problema de investigación. Además, los hallazgos encontrados en la aplicación de la estrategia metodológica sobre el uso de los mapas conceptuales sucesivos depurados (Sanabria et al., 2014) despertaron interés por profundizar en la etapa de planteamiento del problema, surgiendo la necesidad de hacer aportes significativos en esta etapa en dos sentidos: (1) a la estrategia metodológica propuesta en el 2014 sobre el uso de mapas conceptuales sucesivos depurados en los procesos de investigación, y (2) a los cursos de Seminario de investigación. Por lo que, en este estudio se incorporó en la fase de planteamiento del problema una tarea adicional, que consistió en la elaboración de un mapa conceptual sucesivo depurado sobre un tema de interés del maestrante (posible tema de investigación) relacionado directamente con su maestría.

La pregunta orientadora del proceso investigativo de este trabajo fue ¿Cómo pueden ayudar los mapas conceptuales sucesivos depurados a los docentes investigadores a clarificar el problema de su proyecto de investigación?

El diseño de la investigación que aquí se reporta se enmarcó en un enfoque cualitativo, en el que se recurrió al diálogo recursivo (desestabilizador y reflexivo) entre investigadores noveles y expertos. Este diálogo sirvió de vía para facilitarle al investigador novel la depuración de sus mapas conceptuales, por lo que jugó un papel primordial en el proceso desarrollado y el cual fue observado desde distintas perspectivas de análisis.

\section{Investigación Educativa y los Mapas Conceptuales Sucesivos Depurados}

La investigación educativa como todo proceso de investigación busca aportar soluciones o respuestas a problemas o preguntas propias de una situación educativa contextualizada, sin embargo, algunos autores sostienen que esta no impacta en la práctica docente (Perines, 2018). Ante esta realidad y amplio panorama de posibilidades metodológicas y de investigación debe destacarse el papel del docente investigador, como ente activo, reflexivo y observador de su propia práctica como docente, y cómo a partir de esta, logre definir problemas de investigación y encontrar los caminos metodológicos más apropiados para su proceso investigativo. Este autor afirma que uno de los factores que puede ayudar a los docentes investigadores noveles es cambiar la forma en que los investigadores expertos se relacionan con estos docentes, por lo que se propone un proceso de mediación durante la depuración sucesiva de mapas conceptuales. 
Los mapas conceptuales son propuestos por Novak y Gowin (1988), producto de sus investigaciones, con fundamento teórico en la teoría de aprendizaje significativo de Ausubel. Estos autores afirman que el aspecto más relevante de la conducta humana es su formación de conceptos, de representarlos mediante símbolos lingüísticos y manipularlos. Un mapa conceptual es una representación gráfica de conceptos relacionados significativamente por quien lo construye, para formar proposiciones. En la construcción de mapas conceptuales se evidencian los principios del aprendizaje significativo: organización jerárquica de conceptos, su diferenciación y reconciliación integradora. Los autores de este estudio han usado los mapas conceptuales de distintas formas durante los últimos 15 años y han sido facilitadores de talleres con el objetivo de propiciar su uso.

Los mapas conceptuales sucesivos depurados surgen de investigaciones realizadas con docentes para el estudio de temas complejos (Ramírez, Sanabria y Aspée, 2006 y Ramírez, Aspée, Sanabria y Téllez, 2008), con docentes y estudiantes en los procesos de resolución de problemas de Física Universitaria (Ramírez, Sanabria, Aspée y Téllez, 2012 y Ramírez, Sanabria, Téllez, Quintero y Aspée, 2012) y en los procesos de investigación (Sanabria et al., 2014). En estos estudios se encontraron hallazgos favorables en cuanto a los procesos de construcción del conocimiento de quién los construye y depura.

Los mapas conceptuales sucesivos depurados consisten en la reconstrucción de mapas conceptuales por la reflexión de quien los elabora, en la medida que asigna significados a un tema. Como estrategia se usó para facilitar la comprensión de temas complejos (Ramírez, Sanabria y Aspée, 2006) con el propósito de conseguir la borrosidad decreciente de quien aprende. Este proceso reflexivo durante la depuración de los mapas conceptuales es mediado por el diálogo recursivo entre un investigador novel con otro de mayor experiencia.

\section{La estrategia}

La estrategia propuesta es similar a la diseñada en el 2014 (Sanabria et al., 2014) con la diferencia en que se enfoca de forma exclusiva en la fase del planteamiento del problema y se incorpora una tarea adicional que es la elaboración y depuración de un mapa conceptual sobre un tema de su interés (posible tema de investigación relacionado con la maestría). Es decir, durante la elaboración del planteamiento del problema se plantea la elaboración de tres mapas conceptuales y su depuración.

Se partió de las siguientes premisas para su diseño que son producto de la articulación tanto de los hallazgos obtenidos en investigaciones previas (Sanabria et al., 2014) como de los trabajos de Sánchez (2014): (1) Es un proceso complejo, quizás muy complejo, (2) Es cuestión de saberes prácticos y operativos, (3) Se requieren estrategias contextualizadas, (4) Se necesita desarrollar en el investigador novel competencias a partir de quehaceres y prácticas investigativas que influyan en su práctica docente, (5) El tutor/asesor juega un papel primordial en la estimulación del pensamiento crítico, reflexivo y desarrollo de la metacognición del docente investigador novel.

La elaboración de los tres mapas conceptuales se asigna como tarea y se depura cuantas veces sea necesario hasta lograr el consenso entre el participante (informante clave de este estudio) y tutor (o asesor). La depuración se propicia mediante el diálogo recursivo, entre el experto y el novel, con ello se busca "poner a prueba lo que lee o dice desde el punto de vista de la solidez de los razonamientos, de la exactitud de los hechos y la precisión del juicio" (Nussbaum, 2005, p.28). Se buscaba que los investigadores noveles mediante el análisis reflexivo de sus producciones lograran examinarse críticamente al identificar el qué y el cómo de su trabajo.

Con la asignación de tareas (elaboración de mapas conceptuales), se busca que el participante: 
- Exprese sus ideas, conocimientos y comprensión de temas específicos de investigación educativa de su interés y relacionados con la maestría que está cursando

- Plasme los procesos metodológicos de un problema de investigación educativa superando las tradiciones ortodoxas

- Identifique objetos de estudio enmarcados en su contexto y en las líneas de investigación de la maestría

El mapa conceptual de la estrategia se muestra en la Figura 1 y las actividades realizadas por los participantes se describen por tarea a continuación.

Esta tarea consiste en elaborar un mapa sobre los conceptos clave o propios de la maestría con sus posibles temas de investigación, o de interés del participante, y sus alcances. Se recomiendan lecturas de trabajos de grado de maestría y artículos sobre temas relacionados con los posibles conceptos inclusores del mapa. La depuración de este mapa conceptual tiene como objetivo, sin limitarse a ello, que el participante identifique errores conceptuales, inadecuada jerarquización entre conceptos y ambigüedades o confusiones.

Esta actividad tiene como objetivo elaborar un mapa conceptual sobre lo qué es un problema de investigación. La asignación de esta tarea requiere que se haga con anterioridad una sesión con el tutor (asesor o experto) de mayor experiencia, quién expone sus ideas de lo que es un planteamiento del problema, descripción de sus elementos constitutivos, además de facilitar trabajos ya aprobados en el seno de la maestría para su lectura. Con la depuración de este mapa se espera que el participante sea consciente de sus errores epistemológicos, conceptuales y estructurales de un problema de investigación.

En función de los dos mapas conceptuales de las tareas 1 y 2, los participantes deben construir el mapa conceptual de su propio problema de investigación. Este mapa debe contener como mínimo: (a) su objeto de estudio, (b) descripción del contexto de la investigación; (c) rasgos de la situación actual y comparación con el "deber ser", (d) el problema propiamente relacionado con una necesidad, insatisfacción o curiosidad; (e) referentes; (f) posibles soluciones. El proceso de depuración de este mapa conceptual es más complejo y perfila como mínimo la conciencia del participante sobre los aspectos anteriormente señalados.

Durante el diálogo recursivo para la depuración de los mapas surgidos, el tutor/asesor inquiere al participante mediante preguntas recurrentes del tipo: ¿Por qué el tema? ¿Por qué ese y no otro? ¿Qué trabajos ha leído sobre ese tema? ¿Puede identificar o citar algunos referentes, autores o investigadores clásicos o determinantes en esos tópicos? Sobre los conceptos trabajados en el mapa: ¿Es el concepto inclusor, el clave para su posible investigación? ¿Sobre qué o cuales de los conceptos allí plasmados podrías investigar? ¿Sobre qué o quién vas a centrar la observación? ¿Cuál es el problema de investigación? ¿Cuáles sus posibles causas o factores intervinientes? ¿Cuál su posible solución y por qué? 


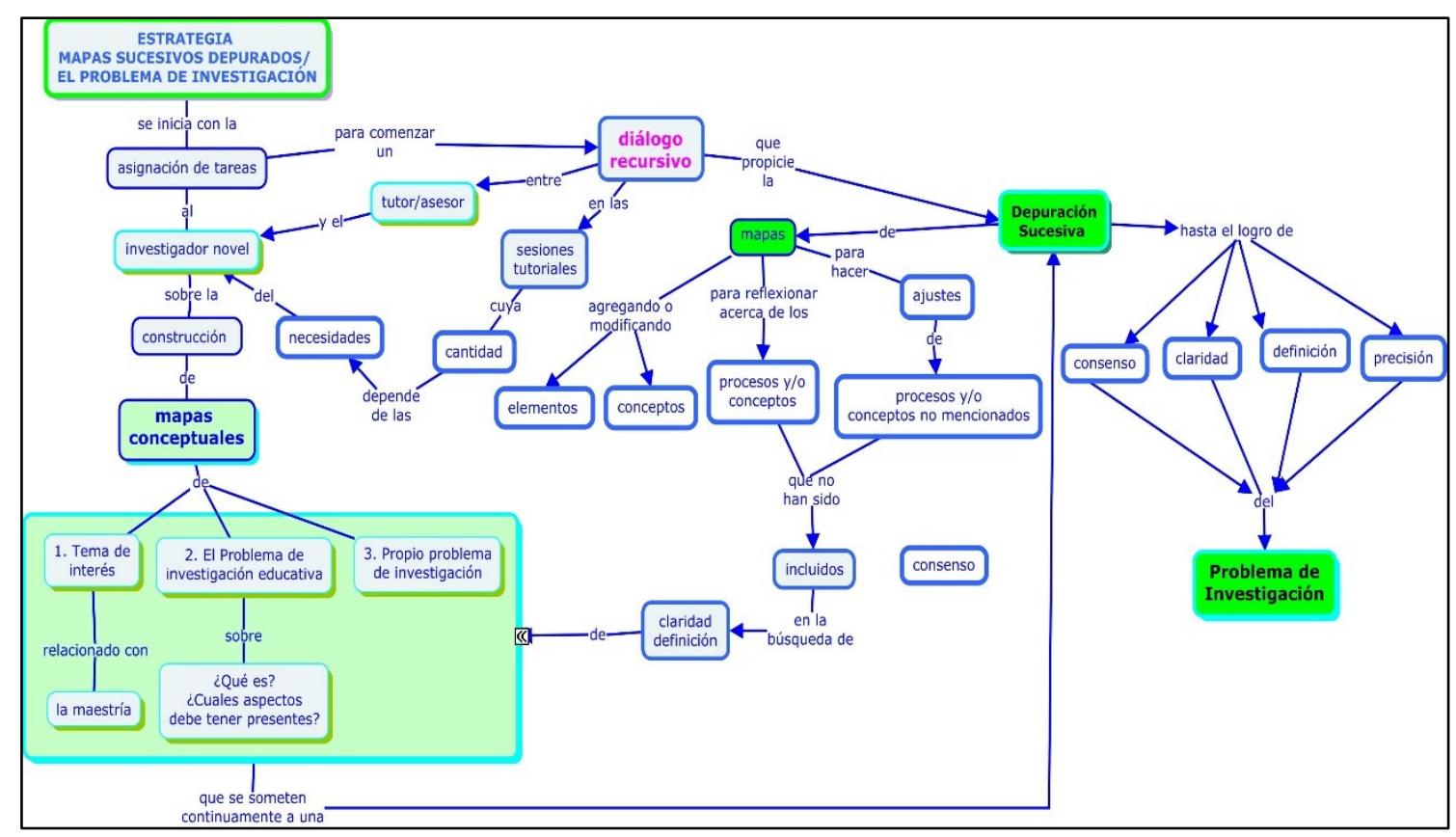

Figura 1. Mapa Conceptual de la estrategia

\section{Marco Metodológico}

La investigación se desarrolló bajo un enfoque cualitativo, nivel descriptivo y un diseño centrado en el método dialógico crítico (Aspée, 2003), en el que se recurre al diálogo recursivo a través de la interacción entre expertos y noveles. Con este diálogo se buscó facilitar, en el investigador novel, la reflexión permanente, la depuración y reconstrucción de los mapas conceptuales elaborados por él sobre: (1) los temas de investigación educativa asociados a la Maestría del informante, (2) problemas de investigación y metodologías usadas en investigación educativa, y (3) sus propios pensamientos y acciones durante el abordaje de su trabajo.

Los participantes se seleccionaron de dos cursos de Seminario de las Maestrías en Innovaciones Educativas y Tecnología Educativa, quienes manifestaron su deseo de participar en el proyecto durante los años 2016 y 2017. La investigación se realizó en la UNET, al interior del Programa la creatividad en la enseñanza de la Física, con cinco profesores noveles en investigación educativa asesorados por la investigadora responsable del proyecto y autora principal de este trabajo

La caracterización de los actores se muestra en la Tabla 1, todos los profesores manejaban adecuadamente los mapas conceptuales y la herramienta CMapTool para la construcción de los mapas conceptuales, la cual es una herramienta informática de libre acceso con fines educativos. Interesó conocer en el contexto de esta investigación, sus experiencias previas, factor influyente en la planificación de las sesiones con cada participante y su necesidad para aclarar sus ideas. 
Tabla 1: Caracterización de Informantes

\begin{tabular}{|c|c|c|}
\hline Informante & Maestría & Descripción \\
\hline I1 & $\begin{array}{l}\text { Innovaciones } \\
\text { Educativas }\end{array}$ & $\begin{array}{l}\text { Profesor de la UNET durante } 6 \text { años. Ingeniero Electricista. Sin experiencia en } \\
\text { Investigación Educativa. Con experiencia en Investigación aplicada en proyectos } \\
\text { técnicos durante } 15 \text { años. }\end{array}$ \\
\hline I2 & $\begin{array}{l}\text { Innovaciones } \\
\text { Educativas }\end{array}$ & Ingeniero Mecánico. Con experiencia de 2 años en docencia universitaria en Física. \\
\hline I3 & $\begin{array}{l}\text { Innovaciones } \\
\text { Educativas }\end{array}$ & $\begin{array}{l}\text { Profesor de Educación Básica. Licenciado en Educación. Sin experiencia en investigación } \\
\text { educativa sólo la adquirida durante su práctica pedagógica durante el pregrado. Con } \\
\text { experiencia } 6 \text { de años como profesor en educación básica. }\end{array}$ \\
\hline I4 & $\begin{array}{l}\text { Innovaciones } \\
\text { Educativas }\end{array}$ & $\begin{array}{l}\text { Profesor del Sistema de Orquestas de Venezuela. Licenciado en Educación, mención } \\
\text { Música. Sin experiencia en Investigación educativa. Con experiencia de } 8 \text { años en } \\
\text { educación básica. }\end{array}$ \\
\hline I5 & $\begin{array}{l}\text { Tecnología } \\
\text { Educativa }\end{array}$ & $\begin{array}{l}\text { Profesor UNET durante } 8 \text { años. Ingeniero Industrial. Sin experiencia en Investigación } \\
\text { Educativa. Con experiencia en Investigación aplicada en proyectos técnicos durante } 5 \\
\text { años (con estudios de Maestría Mantenimiento Industrial). }\end{array}$ \\
\hline
\end{tabular}

Las técnicas usadas fueron la confesión libre y la evocación del recuerdo y los instrumentos se centraron en dos tipos: (1) la grabación de audio de los diálogos entre el experto y el investigador novel durante el proceso de revisión de los mapas conceptuales asignados como tarea y (2) sus producciones, mapas conceptuales construidos por los investigadores noveles y las depuraciones sucesivas de estos mapas.

Se comparten las reflexiones de Martínez (2015) acerca de que "un examen rigurosamente crítico del fundamento de nuestras convicciones, juicios, apreciaciones y creencias reduce el riesgo de error, que es lo máximo a lo que podemos aspirar, pues nunca podremos estar ciertos de haberlo eliminado por completo" (p.84). De allí, que Martínez (2008) se refiera a la validez como la forma de "recoger la información y de las técnicas de análisis que usan. Esos procedimientos los inducen a vivir entre los sujetos participantes en el estudio, a recoger los datos durante largos períodos de tiempo, revisarlos, compararlos y analizarlos de manera continua" (p.67). Los criterios de validez en esta investigación se asocian a que tanto los datos como su análisis, son lo más cónsonos con la realidad vivida por los informantes. Durante el proceso de recogida de datos y análisis de información se corroboraba con los informantes y se contrastaba en función de técnicas e informantes.

La recogida de datos con cada participante varió entre 2 y 4 meses y la planificación de las sesiones dependió de las necesidades de cada informante, en cuanto a periodicidad, duración y actividades. Las sesiones fueron transcritas y codificadas por participante, número de sesión, fecha y duración.

El análisis de resultados se realizó en función de las técnicas de recolección de datos usadas, el proceso de depuración de los mapas conceptuales y el análisis de las interacciones comunicativas de cada participante ocurridas durante el diálogo recursivo con el tutor/asesor. En investigación cualitativa el análisis consiste en la interpretación y reinterpretación de los datos no en su cuantificación, en la búsqueda de conceptos sustantivos a estos datos y sus relaciones (Strauss y Corbin, 2002). Esta etapa requiere de la organización y codificación de los datos, en este estudio se hizo un proceso de codificación abierta, establecida por estos mismos autores como "el proceso analítico por medio del cual se identifican los conceptos y se descubren en los datos sus propiedades y dimensiones" (p.110). 
Se usaron dos categorías iniciales de análisis, ambas se muestran en la Tabla 2. La primera categoría asociada con el análisis de los mapas conceptuales sucesivos depurados, desglosada en las dimensiones propuestas en Sanabria et al. (2014). La segunda categoría construida con base en los estudios de Sharim y Muñoz (2001) quienes, en cuanto a los diálogos verbales, señalan la importancia de relacionar lo que se dice con la situación o el contexto, las características de sus participantes (conocimientos previos, necesidades y propósitos).

Tabla 2: Categorías Iniciales de anàlisis

\begin{tabular}{|c|c|c|}
\hline Categorías & Dimensión & Definición \\
\hline \multirow[t]{5}{*}{ Borrosidad } & Falta de claridad & $\begin{array}{l}\text { Borrosidad en la identificación de conceptos y sus relaciones con otros para } \\
\text { conformar ideas }\end{array}$ \\
\hline & Confusión de conceptos & $\begin{array}{l}\text { Error o equivocación causados por entender, utilizar o tomar un concepto } \\
\text { por otro }\end{array}$ \\
\hline & Ambigüedad & $\begin{array}{c}\text { Conceptos o proposiciones que puede entenderse o interpretarse de } \\
\text { diversas maneras. }\end{array}$ \\
\hline & Concepciones erróneas & Error de significados de un concepto \\
\hline & $\begin{array}{l}\text { Desconocimiento del } \\
\text { tema }\end{array}$ & Incompetencia para asignar significados \\
\hline \multirow{3}{*}{$\begin{array}{l}\text { Diálogo } \\
\text { Recursivo }\end{array}$} & Estructura & Patrones recurrentes \\
\hline & Significado & Construcción y explicitación de los mensajes \\
\hline & Acción & $\begin{array}{c}\text { Organización de las metas e intenciones del hablante y las formas en que el } \\
\text { lenguaje es utilizado al servicio de esa meta }\end{array}$ \\
\hline
\end{tabular}

\section{Resultados}

Los hallazgos se presentan desde dos perspectivas. La primera, por tarea y la segunda por las categorías iniciales y emergentes. Para contextualizar los hallazgos es importante mostrar la cantidad de sesiones y de mapas conceptuales sucesivos depurados generados por Tarea y por Informante (Ver Figura 2).

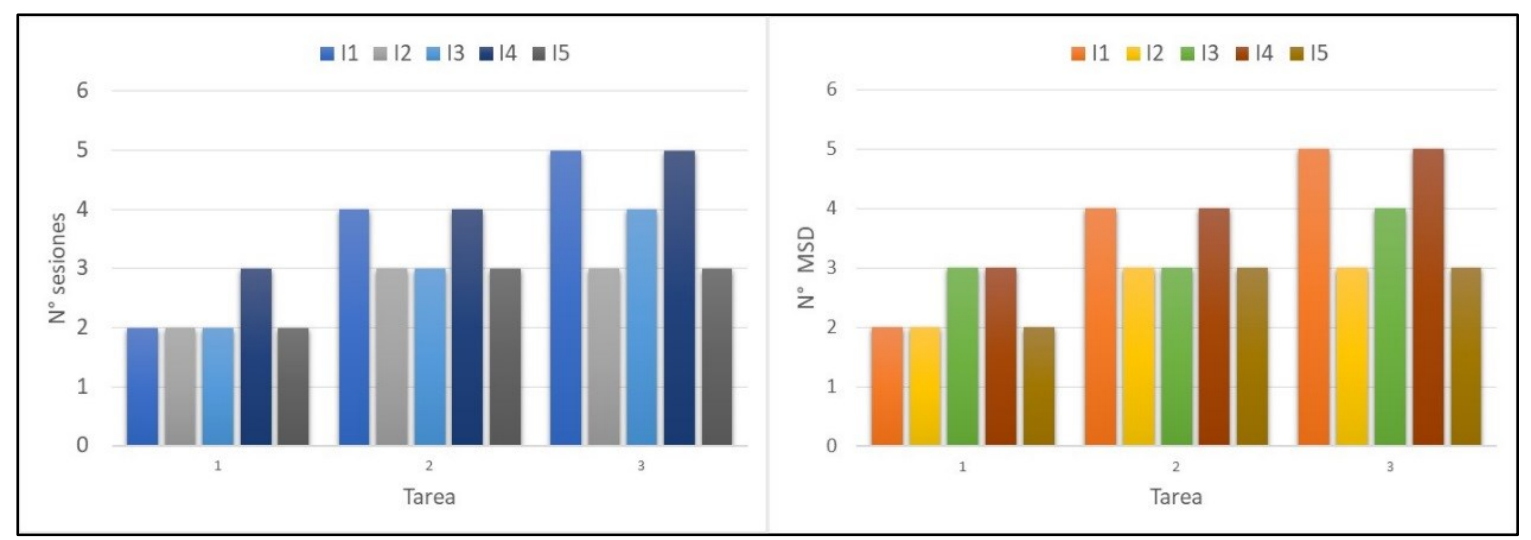

Figura 2: Mapas sucesivos depurados y sesiones por Tarea e Informante 


\subsubsection{Tarea 1}

Esta actividad fue la que menos sesiones de trabajo requirió en todos los participantes, así como la que menor cantidad de mapas conceptuales sucesivos depurados generó en 4 de los 5 informantes. Tres de los cinco (I1, I3 e I4) evidenciaron escasa vinculación entre los conceptos clave o propios de la maestría con sus posibles temas de investigación, así como dispersión, falta de claridad y/o profundidad en el manejo de los conceptos. Fue necesario en los tres informantes mencionados, la recomendación de lecturas asociadas al tema en estudio, lo que les facilitó la depuración del mapa conceptual.

\subsubsection{Tarea 2}

Esta tarea demandó mayor cantidad de sesiones por participante con respecto a la anterior, por lo menos una más. Iguales resultados se presentaron con respecto a la cantidad de mapas conceptuales sucesivos depurados generados. Los informantes I1 e I4 generaron 1 mapa más con respecto a los demás informantes. Todos los participantes construyeron mapas conceptuales sobre esta fase del proceso de investigación, mayormente apoyados en textos especializados.

\subsubsection{Tarea 3}

El inicio de esta tarea tuvo como base los resultados de las dos anteriores. Con respecto a cantidad de tiempo invertido y mapas conceptuales sucesivos depurados generados, fue similar a la Tarea 2 en dos de los informantes (I2, I5). Con los demás informantes (I1, I3 e I4) fue necesario aumentar una sesión y generaron entre uno y dos mapas conceptuales sucesivos depurados más.

Resalta aquí que, todos los participantes presentaron dificultades en la elaboración del mapa de su problema de investigación. Algunos de los participantes (I2 e I5) frecuentemente construían mapas conceptuales, lo que les facilitó que lograran clarificar este mapa en menor tiempo con respecto a los demás participantes. Uno de los informantes (I1) manifestó que el mapa conceptual no le ayudaba en su proceso de investigación y prefería escribir primero segmentos del texto "planteamiento del problema", discutirlo y reflexionar sobre él para luego construir el mapa conceptual, "una vez que en su mente estuviera clarificado su problema de investigación".

\subsubsection{Borrosidad}

En la elaboración de las tres Tareas se evidenció borrosidad en la construcción de los mapas conceptuales la cual disminuyó con los diálogos recursivos y la depuración de los mapas. Cuatro de los cinco participantes evidenciaron desconocimiento de los temas (Tarea 1) asociados a la maestría y dificultad para relacionar el concepto clave con otros conceptos. La Figura 3 muestra parte del mapa conceptual depurado de esta tarea del informante I4, donde se evidencia la complejidad y profundidad del tema abordado: "Evaluación de Entornos Virtuales", ubicado como concepto inclusor, al cual se subordinan conceptos como entorno virtual, evaluación, tipos, ventajas y desventajas, la conectividad, los instrumentos, entre otros. 


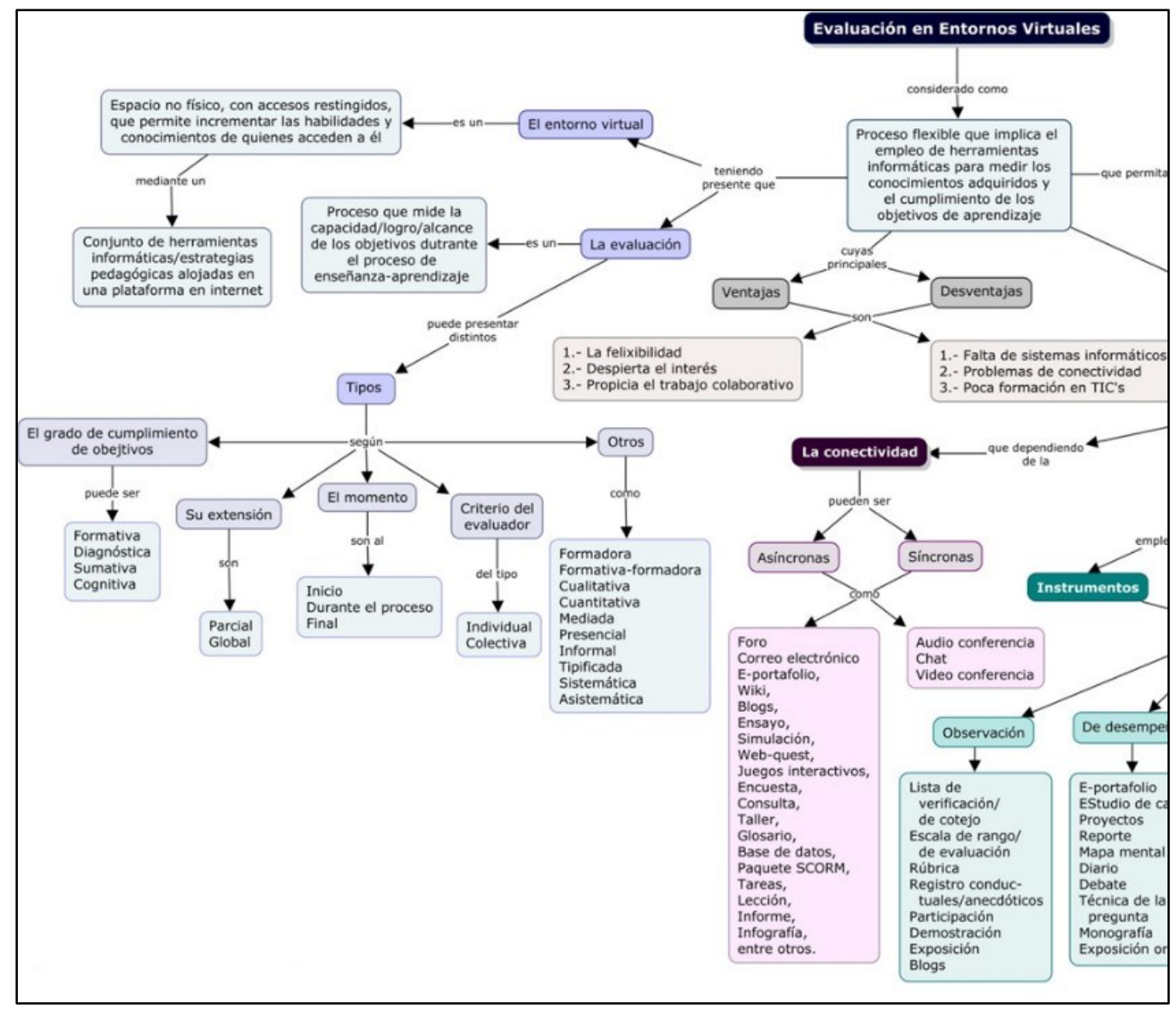

Figura 3. Parte del mapa conceptual sucesivo depurado 2 Tarea 1 del Informante I4.

Sobre la Tarea 2, en el mapa conceptual inicial de todos los informantes se evidenciaron al menos dos dimensiones relacionadas con la variable borrosidad. En tres informantes (I1, I3 e I5) se encontró por lo menos una proposición que carecía de significado coherente. En general, predominó la ambigüedad y el desconocimiento en el manejo de los conceptos asociados a los elementos constitutivos de un problema.

En relación con el mapa conceptual de su propio problema de investigación (Tarea 3) se evidenció mayor borrosidad en todos los participantes, las dificultades encontradas se orientaron a: (a) falta de claridad: no identificaban problemas en sus prácticas docentes o aulas de clase, generalmente piensan que estos están fuera de ellas, en la institución, el entorno social, el país, entre otros, (b) confusión de conceptos: entre el problema en estudio y los problemas subordinados a él, entre objetivos y actividades para el logro de los objetivos, (c) ambigüedad: entre el objeto de estudio y el problema, (d) concepciones erróneas: no existía correspondencia entre las interrogantes de la investigación y los objetivos, formulación errónea de objetivos de investigación, los objetivos específicos no apuntan al logro del objetivo general, (f) desconocimiento: del contexto en el que surge el problema a investigar, sobre referentes del tema objeto de estudio, de los principales hallazgos o posibles soluciones encontradas en estudios previos relacionados, ( $\mathrm{g}$ ) abordaje inalcanzable de la investigación por distintas causas: grado de complejidad, tiempo previsto, complejidad del tema, multiplicidad de factores intervinientes y dificultad para jerarquizarlos.

La Figura 4 muestra el segundo mapa conceptual sucesivo depurado por el Informante 4 de la Tarea 3, con tres cuadros con observaciones y preguntas del tutor/asesor al docente investigador. En la figura se muestran las siguientes interrogantes: ¿Diseño de qué? ¿Metodológico? ¿A qué dinámicas se refiere? 
buscan clarificar y precisar el abordaje del tema en estudio. También se presentan observaciones sobre la ambigüedad del problema y objeto de estudio.

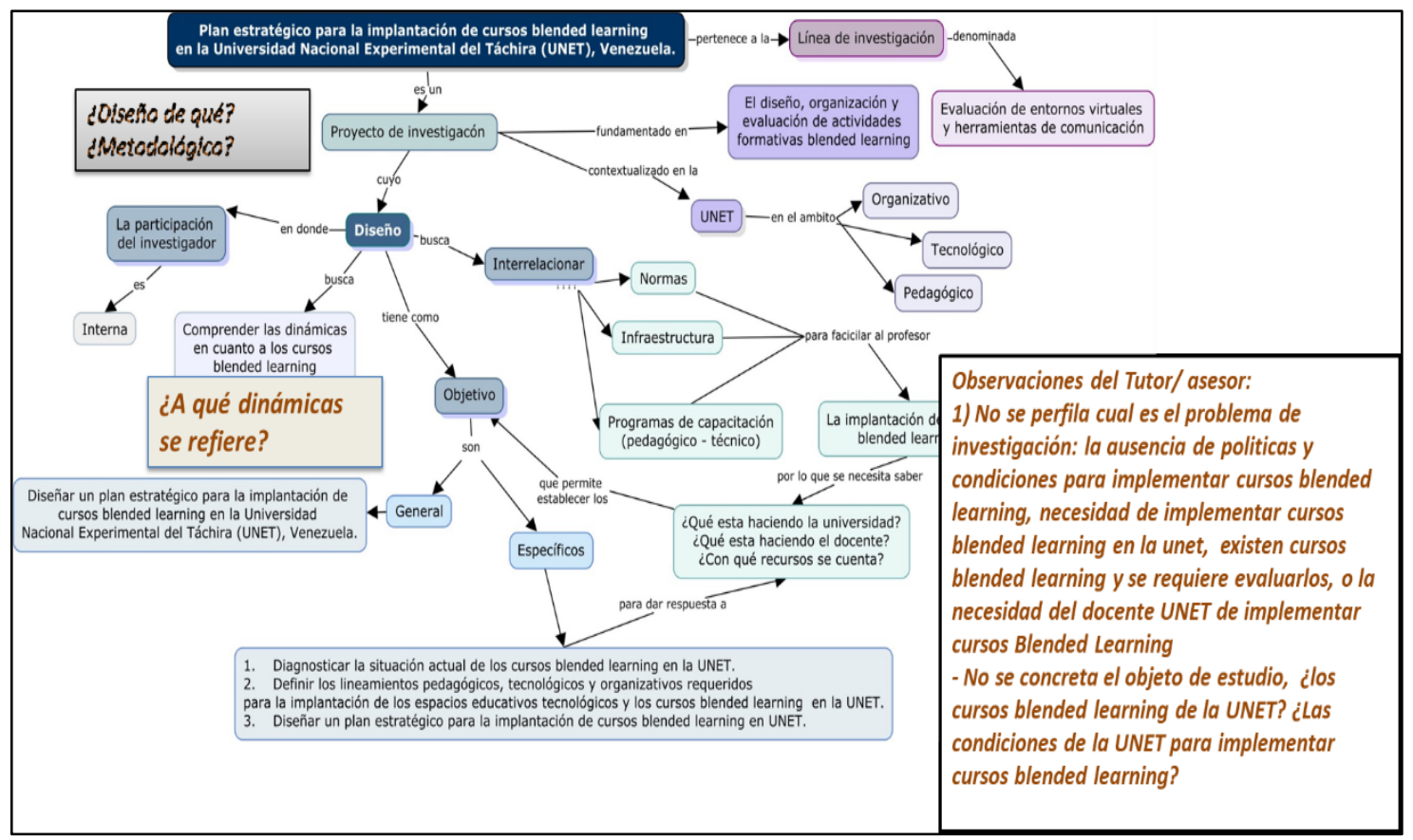

Figura 4. Mapa Conceptual Sucesivo Depurado 2 Tarea 3 del Informante I4.

\subsubsection{El diálogo recursivo}

El diálogo recursivo se observó desde tres dimensiones, la estructura, el significado de los mensajes y la acción de los participantes en este diálogo. La estructura se refiere a los patrones recurrentes durante la interacción (Sharim y Muñoz, 2001) evidenciado durante las sesiones de depuración de los mapas conceptuales. Sobre esta dimensión se encontró:

- La actitud de los informantes en la primera sesión de cada tarea fue de defensa a las ideas expresadas en los mapas conceptuales, en la medida que avanzaba el diálogo asumían posturas de cuestionamiento que disminuía a medida que el tutor usaba recursos para activar procesos mentales de comprensión.

- Dos de los informantes (I1, I3 e I5) no tomaron decisiones por estados emocionales de inacción en las sesiones iniciales de depuración de los mapas conceptuales de las distintas tareas. Las palabras de aliento del tutor/asesor propiciaron actitudes positivas en estos informantes a medida que disminuyó su borrosidad acerca del tema tratado en el mapa conceptual.

- La intencionalidad del experto en sus intervenciones buscaba desestabilizar las posturas del investigador novel en todas las sesiones. Se evidenciaron estados emocionales de inquietud (o angustia) en los investigadores noveles, sin embargo, fueron adquiriendo mayor confianza para expresar sus ideas en la medida que reflexionaban sobre ellas.

- Los significados construidos por los informantes fueron más complejos durante la depuración del mapa conceptual sobre "su problema de investigación" y menos complejo en la Tarea 1. 
Estos hallazgos se corresponden con los expresado por Sharim y Muñoz (2001) sobre la "propiedad del discurso el logro de una acción, que emerge no del arreglo de las unidades subyacentes sino de la organización de las metas e intenciones del hablante y de las maneras en que el lenguaje es utilizado al servicio de esa meta" (p. 305)

En la Tarea 2 (qué es un problema de investigación) se evidenció en todos los participantes el manejo de algunos conceptos de forma desarticulada y sin conexión significativa con los conceptos presentados en su mapa conceptual. El diálogo evidenció que la mayoría de los participantes pasaron de la repetición verbal del texto metodológico de apoyo, al manejo y apropiación del discurso metodológico, se evidenció además que los investigadores noveles lograron cohesionar lo presentado en el texto de apoyo y sus concepciones acerca de la etapa de planteamiento del problema. Esto se logra, al articular la información con su propia experiencia como docente lo que se compadece con lo expresado por Perines (2018). Todo esto dio muestras que la comprensión de los conceptos abordados en el mapa, y la construcción y reconstrucción de significados fue facilitada por el diálogo recursivo (novel tutor/asesor), sobre todo por la complejidad que representó cohesionar los conceptos en el mapa conceptual y el manejo del lenguaje propio de los problemas de investigación.

En relación con el propio problema de investigación (Tarea 3), todos los participantes evidenciaron problemas en cuanto a la (a) identificación de conceptos clave, (b) organización de conceptos para formar significados coherentes y lógicos, (c) integración de conceptos relevantes para sus investigaciones y (d) adecuación de conceptos de acuerdo con el tema de investigación abordado. Se puso de manifiesto una construcción intersubjetiva de significados (entre asesor y novel) que permitió la creación de un marco común de lenguaje propio del proyecto de investigación del informante, lo que contrasta con lo señalado por Perines (2018) cuando afirma que "la ausencia de un vocabulario común obstaculiza la capacidad de los futuros docentes para acceder a un corpus de conocimiento sobre la enseñanza y la investigación" (p. 13). En todos los participantes se manifestó un desarrollo de la habilidad para articular y cohesionar los conceptos a medida que avanzó la experiencia.

La acción de los participantes, tanto del experto como los noveles investigadores, durante el diálogo en todos los casos fue proactiva en la búsqueda de consensos y caminos de orientación del trabajo de investigación. Sin embargo, se destaca la fluidez de los diálogos (ciclos de Acción-ReacciónRetroalimentación), a pesar de que algunos participantes no manejaban los conceptos asociados a cada una de las tareas de forma adecuada, ni con la profundidad requerida.

El diálogo recursivo y la elaboración de mapas conceptuales sucesivos depurados, en tres de los informantes (I1, I3 e I5), facilitó la reflexión acerca del problema de investigación, el acercamiento de forma crítica a su objeto de estudio, esto mejoró su comprensión y les permitió perfilar posibles caminos de solución. Sólo dos de los informantes (I2 e I4) tenían mayor claridad en su objeto de estudio, de lo que querían investigar y la depuración de sus mapas les dio indicios sobre las orientaciones metodológicas a seguir y la construcción de sus objetivos de investigación.

Se destaca como hallazgo importante el compromiso, la motivación y el desarrollo de una actitud reflexiva y crítica en los participantes hacia su investigación, aspecto considerado clave para facilitar la construcción de conocimientos y su crecimiento como investigador.

\subsubsection{Categoría Emergente: Estados Emocionales}

Del análisis de la información recabada de los informantes surgió, de forma reiterada, una serie de aspectos interesantes, por lo que se consideró apropiado organizarlos en la categoría estados emocionales para su descripción y análisis como categoría emergente, factor estudiado también por Ochoa (2011). 
En todas las actividades (Tareas), todos los participantes manifestaron incertidumbre, dudas y cierta intimidación al ser cuestionados sobre lo plasmado en sus mapas. Los diálogos relacionados con la Tarea 1 generaron en 3 de los participantes (I1, I3 e I5) sorpresa e incredulidad al hacer conciencia sobre las ambigüedades, errores y escasa profundidad en el manejo de los conceptos asociados a la maestría.

La construcción del mapa conceptual de su propio problema de investigación (Tarea 3) generó más angustia e inquietud en los participantes, la cual disminuyó en la medida que se clarificaba el problema de investigación y sus elementos constitutivos. Todos enfrentaron situaciones de ansiedad por las confusiones y ambigüedades evidenciadas en esta etapa, condiciones que fueron superadas por el acompañamiento del tutor/asesor y la borrosidad decreciente evidenciada durante la depuración sucesiva del mapa.

En tres informantes (I1, I3 e I5) se evidenció algún grado de bloqueo y negación que no les permitió avanzar de la forma esperada en las primeras sesiones de esta tarea, a consecuencia de la complejidad del problema de investigación, según lo expresado por ellos mismos. I3 e I5 manifestaron que el tiempo dedicado a la reflexión sobre el problema de investigación fue excesivo y poco fructífero, consideración que cambió al avanzar en la depuración de sus mapas. Los participantes I2 e I4 reflejaron mayor estabilidad emocional por la claridad que tenían sobre su tema de investigación y mostraron apertura hacia las sugerencias del tutor/asesor durante el desarrollo de la experiencia. Estos resultados se corresponden con lo expresado por Ochoa y Cueva (2017) en cuanto a los bloqueos experimentados por los tesistas de maestrías durante su proceso investigativo.

Las preguntas realizadas por el tutor/asesor en todas las sesiones desestabilizaron emocionalmente a los informantes generándoles conflictos cognitivos (manifestados como dudas o confusión). De forma progresiva ellos tomaron conciencia de lo importante del manejo adecuado de sus emociones, y tornaron positivos sus estados emocionales de incertidumbre e inquietud, esto se reflejó en todos los participantes como una actitud proactiva hacia la reflexión sobre su propio accionar como docente e investigador.

\section{A manera de Conclusiones}

El planteamiento del problema en los docentes investigadores noveles es un problema recurrente en las maestrías sobre educación, motivo por el cual se diseñó e implementó una estrategia con el propósito de ayudar a estos docentes a clarificar su problema de investigación educativa mediante el uso de los mapas conceptuales sucesivos depurados y la mediación de un experto/tutor en investigación facilitada por un diálogo recursivo. Se corrobora el alto grado de borrosidad en los docentes noveles investigadores cuando inician su proceso investigativo, esta borrosidad es asociada a la falta de claridad en su tema de investigación y objeto de estudio, la confusión de conceptos sobre los posibles de temas de investigación y el lenguaje propio de los problemas de investigación, la ambigüedad con la que maneja conceptos claves de investigación educativa, los propios de educación y práctica docente, las concepciones erróneas y en algunos casos intuitivas sobre investigación y educación, y desconocimiento de temas relacionados con la maestría e investigación educativa.

Juega un papel primordial en este estudio la figura del tutor, asesor o experto, quien, a través del diálogo recursivo, mantiene interacción con el participante (docente e investigador novel) provocando, en él, de forma intencional un proceso reflexivo y metacognitivo sobre su propia investigación y sobre los posibles caminos metodológicos para abordarla. Los diálogos recursivos entre docentes noveles investigadores y experto/tutor o asesor durante la depuración de mapas conceptuales propician la disminución de los rasgos de borrosidad existentes en ellos. 
Un hallazgo importante se asocia al estado emocional de los participantes durante la problematización y su influencia en el avance de su proceso. Los estados de parálisis o negación son superados por los participantes con la orientación del tutor, quien media para que los participantes logren hacerse conscientes de la necesidad de manejar de forma apropiada sus emociones.

La estrategia metodológica Mapas Conceptuales Sucesivos Depurados/Problema de Investigación resulta promisoria para clarificar y concretar problemas de investigación. Su implementación requiere de inversión de tiempo y dedicación por parte de los actores (investigadores noveles y expertos), por lo que su incorporación en cursos de Seminario de Investigación debe ser estudiada y considerada. Si este es el caso, es necesario un replanteamiento de estos cursos en la búsqueda de mayor flexibilidad, contextualización y adaptación a las necesidades de los docentes que recién ingresan en el mundo de la investigación educativa.

Para futuros trabajos se propone estudiar cómo se relaciona la construcción de los mapas conceptuales con la escritura académica del planteamiento del problema.

\section{Referencias Bibliográficas}

Aspee, M. (2003). La metacognición en los tiempos del caos. Tesis doctoral. Caracas: Universidad Santa María.

Martínez, M. (2008). Epistemología y metodología cualitativa en las ciencias sociales. Ciudad de México: Trillas.

Martínez, M. (2015). Comportamiento humano: nuevos métodos de investigación. $2^{\text {a }}$ edición, Ciudad de México: Trillas.

Moreno, M. (2016). La formación de nuevos investigadores educativos. Revista de la educación superior, 45(177), 171-175. Disponible en: https://dx.doi.org/10.1016/j.resu.2016.04.005.

Najera, F., Murillo, R., \& García, G. (2017). Experiencia en la formación de investigadores educativos en posgrado, a través de la tutoría. En L. A., Acuña, A., Barraza y A., Jaik, (Coord). Formación de investigadores educativos en Latinoamérica: hacia la construcción de un estado del arte, 151-169. México: Redie.

Nussbaum, M. (2005). El cultivo de la humanidad. Una defensa clásica de la reforma en la educación liberal. Barcelona: Paidós.

Novak, J., y Gowin, D. (1988). Aprendiendo a Aprender. Barcelona: Martínez Roca.

Ochoa, L. (2011). La elaboración de una tesis de maestría: exigencias y dificultades percibidas por sus protagonistas, Entornos, (24), 171-183. Recuperado de: https://www.google.com/url?sa $=t \& r c t=j \& q=\& e s r c=s \&$ source $=$ web $\& c d=1 \& c a d=r j a \& u a c t=8 \& v e d=2$ ahUKEwiHrKPIOKjmAhUhwFkKHY 9DA0oQFjAAegQIBBAC\&url=https\%3A\%2F\%2Fdialnet.unirioja.es\%2Fdescarga\%2Farticulo\%2F3798839 .pdf\&usg=AOVVaw3esThiJoHEksqOG4SDWhPE 
Ochoa, L., y Cueva, A. (2017). El bloqueo en el proceso de elaboración de una tesis de maestría; angustias y desazones percibidas por sus protagonistas. Revista Lenguaje, 45(1), 61-87.

Ochoa, L., y Cueva, A. (2012). Tesis y deserción entre el compromiso y el obstáculo. Bogotá: Universidad Nacional de Colombia.

Perines, H. (2018). ¿Por qué la investigación educativa no impacta en la práctica docente? Estudios sobre Educación. Revista Semestral de la Facultad de Educación y psicología de la Universidad de Navarra, 34, 9-27.

Ramírez de M., Sanabria, I., y Aspée, M. (2006). El control metacognitivo de la borrosidad decreciente en la elaboración de mapas conceptuales. En Cañas, A., y Novak, J. (Eds). Second International Conference on Concept Mapping, 1, 99-207.

Ramírez de M. M., Aspée M., Sanabria I., y Téllez N. (2008). El Control Metacognitivo y los Mapas Conceptuales para facilitar la Comprensión de Estructuras Complejas. Revista Científica UNET, 20(1), 5161.

Ramírez de M., M., Sanabria, I., Aspee, M., y Téllez, N. (2012). Resolución de problemas de física: averígüese con mapas metacognitivos lo que el profesor ya sabe...y que aprenda en consecuencia. En Cañas, A., Novak, J., Vanhear, J., (Eds). Fifth International Conference on Concept Mapping, 2, 382-389.

Ramírez de M., M., Sanabria, I., Téllez, N., Quintero A., y Aspée, M. (2012). El Mapa Metacognitivo para organizar y orientar la Resolución de Problemas. Memorias del Congreso Regional de Investigación Educativa. UCAT: San Cristóbal.

Sanabria, I., Ramírez de M., M., Téllez, N., y Aspée, M. (2014). Mapas Conceptuales Sucesivos Depurados para Facilitar un Proceso de Investigación. En Correia, P., Malachias, M., Cañas, A., y Novak, J. (Eds). Sixth International Conference on Concept Mapping, 1, 338-445.

Sánchez, R. (2014). Enseñar a Investigar. Una didáctica nueva de la investigación en ciencias sociales y humanidades. Ciudad de México: Universidad Nacional Autónoma de México e Instituto de Investigaciones sobre la Universidad y la Educación.

Sharim, S., y Muñoz, D. (2001). Bases Teóricas para el estudio de la interacción verbal. Universidad de Chile. BFUCh, XXXVIII (2000-2001), 301 -327.

Strauss, A., y Corbin, J. (2002). Bases de la investigación cualitativa: técnicas y procedimientos para desarrollar la teoría fundamentada. Medellín: Universidad de Antioquia.

Valarino H., E. (1997). Enseñar a Investigar contra el Síndrome TMT. Trabajo de ascenso para profesor titular. Caracas: Universidad Central de Venezuela. 